\title{
Associação entre fatores nutricionais e o controle glicêmico de crianças e adolescentes com diabetes melito tipo 1
}

\author{
Relationship between nutrition factors and glycemic control \\ in children and adolescents with type 1 diabetes mellitus
}

Karla Cristina Queiroz', Ivani Novato Silva², Rita de Cássia Gonçalves Alfenas ${ }^{3}$

\begin{abstract}
RESUMO
Objetivo: Identificar fatores nutricionais que influenciaram o controle glicêmico da população estudada. Sujeitos e métodos: Participaram do estudo 146 crianças e adolescentes, entre 7 e 19 anos de idade, atendidos na Divisão de Endocrinologia Pediátrica do Hospital das Clínicas da Universidade Federal de Minas Gerais. A dieta foi avaliada por meio de Questionário Quantitativo de Frequência Alimentar validado e adaptado à população estudada. $O$ controle glicêmico foi avaliado como bom, regular ou ruim a partir da média de dois resultados de $\mathrm{HbA} 1 \mathrm{c}$ dos seis meses anteriores à data da avaliação dietética. Resultados: Dietas com maior teor de proteína, menor teor de gordura saturada e com índice glicêmico (IG) e carga glicêmica (CG) mais baixos afetaram positivamente o controle glicêmico dos indivíduos estudados. 0 hábito de consumir sacarose e merenda gratuita influenciou negativamente o controle glicêmico. Conclusão: O consumo de dietas nutricionalmente adequadas e de baixo IG/CG favoreceu o controle glicêmico da população estudada. Arq Bras Endocrinol Metab. 2010;54(3):319-25
\end{abstract}

\section{Descritores}

Diabetes melito tipo 1; consumo de alimentos; dieta para diabéticos; glicemia
1 Hospital das Clínicas, Faculdade de Medicina, Universidade Federal de Minas Gerais (UFMG), Belo Horizonte, MG, Brasil ${ }^{2}$ Departamento de Pediatria, Faculdade de Medicina, UFMG, Belo Horizonte, MG, Brasil ${ }^{3}$ Departamento de Nutrição e Saúde, Universidade Federal de Viçosa (UFV), Viçosa, MG, Brasil

\begin{abstract}
Objective: To identify nutritional factors that affected the glycemic control of the studied population. Subjects and methods: One hundred forty-six children and adolescents, aged 7 to 19 years, followed-up at the Division of Pediatric Endocrinology of the Hospital das Clínicas da Universidade Federal de Minas Gerais participated in the study. The diet of each participant was evaluated by means of a Quantitative Food Frequency Questionnaire, previously validated in a pilot-project. Glycemic control was assessed through the average of $\mathrm{HbA} 1 \mathrm{c}$ results obtained six months prior to date of diet evaluation. Results: Diets with high protein content, less saturated fats, and lower glycemic index (GI)/glycemic load (GL) had a positive effect on glycemic control. The consumption of sucrose and of free snacks provided by the school had a negative influence on glycemic control. Conclusion: The consumption of nutritionally adequate and low GI/GL diets favored glycemic control of the studied population. Arq Bras Endocrinol Metab. 2010;54(3):319-25
\end{abstract}

Correspondência para: Ivani Novato Silva Divisão de Endocrinologia Infantil e do Adolescente, Hospital das Clínicas, Departamento de Pediatria, Faculdade de Medicina, UFMG Av. Alfredo Balena, 190, sala 267 30130-100 - Belo Horizonte, MG, Brasil

ivanins@medicina.ufmg.br

Recebido em 5/Ago/2009 Aceito em 18/Jan/2010

\section{Keywords}

Diabetes mellitus type 1; food consumption; diabetic diet; blood glucose

\section{INTRODUÇÃO}

$\mathrm{O}$ diabetes melito tipo 1 (DMl) é o distúrbio endocrinometabólico mais frequente na infância e na adolescência e apresenta incidência crescente com alta morbimortalidade e perda da qualidade de vida de seus portadores $(1,2)$.
Estudos clínicos e epidemiológicos demonstram associação entre as complicações do DM e controle glicêmico inadequado: no Diabetes Control and Complications Trial (DCCT) foi demonstrado que o controle glicêmico adequado retarda o início e a progressão das complicações microvasculares, bem como reduz o risco 
de qualquer evento cardiovascular em $42 \%$ e de infarto do miocárdio não fatal, acidente vascular cerebral e morte em $57 \%(3,4)$.

O objetivo principal do tratamento do DM é a obtenção de um bom controle metabólico, necessário para o crescimento e desenvolvimento adequados da criança, para minimizar o risco das complicações crônicas e melhorar a qualidade de vida dos pacientes. O plano ideal de tratamento para crianças e adolescentes com DMl integra a terapia insulínica com a dieta e atividade física. O tratamento deve ser individualizado e as metas devem ser estabelecidas pelos profissionais de saúde em conjunto com o paciente e/ou responsáveis (5).

A adoção de hábitos alimentares adequados é fundamental para o controle do DM e, consequentemente, para a prevenção das complicações agudas e crônicas da doença. O carboidrato tem sido apontado como o componente da dieta que tem maior influência na glicemia. A quantidade de carboidrato ingerida é usualmente o principal determinante da glicemia pós-prandial, mas o tipo de carboidrato ingerido também pode afetar essa resposta (5). Os hábitos alimentares estão relacionados à cultura e diferem entre as populações.

Nas principais bases de dados de pesquisas, não foram encontradas publicações nacionais com relatos da influência da dieta no controle glicêmico de crianças e adolescentes com DMl. Estudos dessa natureza são importantes, pois permitem identificar as principais inadequações nutricionais e sua relação com o controle glicêmico. Além disso, servem de subsídio para o planejamento de intervenções nutricionais direcionadas, que poderão contribuir para o melhor controle metabólico do paciente diabético. Este estudo teve como objetivo identificar fatores nutricionais que influenciaram o controle glicêmico da população estudada.

\section{SUJEITOS E MÉTODOS}

\section{Casuística}

Participaram do estudo crianças e adolescentes com DMl, na faixa etária de 7 a 19 anos, com no mínimo um ano de diagnóstico da doença, atendidos na Divisão de Endocrinologia Pediátrica do Hospital das Clínicas da Universidade Federal de Minas Gerais (HC-UFMG). O cálculo do tamanho da amostra mínima ( $\mathrm{n}=140)$ para o atual estudo foi estabelecido considerando o número total de crianças e adolescentes com DMl $(\mathrm{n}=240)$ que atendiam aos critérios anterior- mente descritos e perda amostral de 10\%. Foi adotada margem de erro de $5 \%$, nível de confiança de $90 \%$ e variância máxima para as respostas de proporção. A estratégia amostral foi determinada por recenseamento, isto é, foram entrevistados os indivíduos que frequentaram o ambulatório do HC-UFMG no período de junho de 2007 a março de 2008 , em dias alternados e aleatórios.

O estudo foi aprovado pelo Comitê de Ética da Universidade Federal de Minas Gerais. Os pais ou responsáveis ou o próprio entrevistado, quando maior de 18 anos, assinaram o termo de consentimento, após serem esclarecidos sobre os objetivos da pesquisa.

\section{Métodos}

Os dados foram obtidos por meio de um questionário semiestruturado previamente testado em projetopiloto, respondido pelos responsáveis ou pelos próprios pacientes. Além disso, foram realizadas pesquisas em prontuários médicos.

Os indivíduos foram agrupados em três faixas etárias: 7 a 10 anos, 11 a 14 anos e 15 a 19 anos. A renda familiar e per capita foi avaliada em relação ao saláriomínimo (SM), que na ocasião da entrevista correspondia a R\$ 380,00 (US\$ 194,00).

Foram aferidos dados de peso e estatura de acordo com as técnicas preconizadas por Jellife (6) e calculouse o índice de massa corporal (IMC) dividindo-se o peso em quilogramas, pela estatura em metros ao quadrado (7). A partir do cálculo do IMC, as crianças e os adolescentes foram classificados quanto ao estado nutricional, de acordo com os pontos de corte atualmente recomendados em (8): baixo peso $(<$ percentil $5)$, eutrofia ( $\geq$ percentil 5 e $<$ percentil 85 ), sobrepeso ( $\geq$ percentil 85 e $<$ percentil 95 ) e obesidade ( $\geq$ percentil 95). Os percentis foram gerados pelo programa DietPro - versão 4(9), que utiliza a referência antropométrica do Center for Disease Control and Prevention (CDC, 2000).

O controle glicêmico foi avaliado pela média de dois resultados de hemoglobina glicada $(\mathrm{HbAlc})$ dos seis meses anteriores à data da avaliação dietética, obtidos em prontuário, a partir das consultas realizadas trimestralmente. Foi classificado, de acordo com o proposto por Chase, em bom quando a relação entre a HbAlc e o valor limite superior de normalidade do método era $<1,33$, regular se $\geq 1,33$ e $<1,5$ e ruim se $\geq 1,5(10)$.

A ingestão habitual dos pacientes nos seis meses precedentes à entrevista foi analisada por meio de um questionário quantitativo de frequência alimentar 
(QQFA) validado (11). Foi utilizado registro fotográfico, contendo fotos de porções pequena, média e grande, para favorecer o aumento da precisão na estimativa das porções dos alimentos ingeridos (12). A composição nutricional das dietas ingeridas foi analisada utilizando o software DietPro ${ }^{\circledR}(9)$. A composição química dos alimentos incluídos no QQFA foi cadastrada no software, considerando-se preferencialmente os dados da Tabela Brasileira de Composição de Alimentos (13). Informações complementares foram retiradas de outras duas tabelas $(14,15)$. A avaliação dietética incluiu ainda uma anamnese alimentar sobre o número e os horários das refeições e lanche escolar. Avaliou-se a relação entre a composição nutricional, o padrão alimentar e o controle glicêmico da população estudada.

Os alimentos foram divididos em sete grupos seguindo a recomendação da pirâmide alimentar americana adaptada à população brasileira, e as guloseimas e as gorduras foram agrupadas em um único grupo devido à pequena participação destes no consumo alimentar total (16). Avaliou-se a participação relativa desses grupos em relação ao consumo total de alimentos, mediante a divisão do consumo médio de cada grupo pelo somatório do consumo médio de todos os grupos (em gramas e/ou mililitros). Em seguida, a participação relativa dos grupos de alimentos no consumo alimentar total foi avaliada em relação ao controle glicêmico dos indivíduos estudados.

A dieta habitualmente ingerida por cada participante foi analisada quanto ao índice glicêmico (IG), utilizando equação descrita por Wolever e Jenkins (17), recomendada pela FAO (1998). O IG de cada alimento foi obtido considerando a glicose como referência, utilizando os valores publicados na Tabela Internacional de Índice Glicêmico (18). Para alimentos cujo IG não foi indicado nesta tabela, foi considerado o IG de outros alimentos que apresentavam composição nutricional e modo de preparo semelhante. A carga glicêmica (CG) foi estimada utilizando a equação proposta por Foster-Powell e cols. (18).

\section{Análise estatística}

Os softwares "Statistical Package for the Social Sciences for Windows", versão 13.0 (SPSS), e "R Project of Statistical Computing", versão 2.6.1, foram usados para análise dos dados. Para avaliar a associação entre variáveis categóricas, utilizou-se o teste Qui-Quadrado ou o teste Exato de Fisher, quando indicado. Para com- parar variáveis numéricas entre grupos independentes, foi adotado o teste T-Student e o teste ANOVA. Por meio do teste de Tukey, foram realizadas comparações múltiplas entre os grupos, com objetivo de identificar as diferenças. Para todos os testes, adotou-se o nível de significância de $5 \%$.

\section{RESULTADOS}

Na tabela 1 , são apresentadas as características clínicas e demográficas dos participantes do estudo. Foram estudados 146 diabéticos, com predominância do sexo feminino $(62,3 \%)$. Das 130 famílias avaliadas, 96,2\% possuíam renda de até cinco salários-mínimos (SM) e $62,3 \%$, de até dois SM, caracterizando uma população de baixo poder aquisitivo, sendo que $38,5 \%$ das mães e $34,3 \%$ dos pais estudaram até quatro anos. A dose média de insulina (NPH + regular ou ultrarrápida) aplicada pelos pacientes foi $0,84 \pm 0,2 \mathrm{U} / \mathrm{kg}$. Dos 143 indivíduos avaliados, $80,8 \%$ aplicavam insulina conforme prescrição médica. A maioria dos entrevistados $(91,6 \%)$ utilizava múltiplas doses de insulina ajustada conforme glicemias pré-prandiais. Vinte e um pacientes (16\%) relataram aplicar quatro doses de insulina/dia (2 de NPH e 2 de regular), $36(27,2 \%)$, cinco doses ( 2 de NPH e 3 de regular) e $74(56,8 \%)$, seis doses ( 3 de NPH e 3 de regular). Apenas um paciente fazia o ajuste da dose de insulina de acordo com a quantidade de carboidratos ingeridos.

Tabela 1. Características clínicas e demográficas da população estudada

\begin{tabular}{|c|c|}
\hline Variáveis & Média \\
\hline Idade média (anos) & $12,9 \pm 3,6$ \\
\hline Sexo feminino (\%) & 62,3 \\
\hline Escolaridade paterna (anos) ${ }^{\mathrm{a}}$ & $6,64 \pm 3,5$ \\
\hline Escolaridade materna $(a n o s)^{b}$ & $6,88 \pm 3,4$ \\
\hline Renda per capita $(\mathrm{R} \$)^{c}$ & $201,00 \pm 153,00$ \\
\hline Tempo médio de doença (anos) & $6,9 \pm 3,6$ \\
\hline Idade média ao diagnóstico (anos) & $6,0 \pm 3,4$ \\
\hline Dose de insulina $(\mathrm{U} / \mathrm{kg})^{d}$ & $0,84 \pm 0,2$ \\
\hline Baixo peso (\%) & 8,9 \\
\hline Sobrepeso (\%) & 3,4 \\
\hline Eutróficos (\%) & 87,7 \\
\hline Controle glicêmicoa bom (\%) & 38,1 \\
\hline Controle glicêmicoa regular (\%) & 18,0 \\
\hline Controle glicêmicoa ruim (\%) & 43,9 \\
\hline
\end{tabular}

a: $n=139 ; b: n=141 ; c: n=130 ; d: n=143$. 
Bom controle glicêmico foi observado em 38,1\% dos indivíduos. Quando os pacientes foram agrupados de acordo com a faixa etária, houve diferença estatisticamente significante no grau de controle entre os grupos com 7 a 10 e 15 a 19 anos $(\mathrm{p}=0,025)$. Os grupos de 7 a 10 anos e de 11 a 14 anos apresentaram controle glicêmico regular, com média do índice adotado para classificação de $1,37 \pm 0,28$ e $1,51 \pm 0,4$, respectivamente. Já no grupo de 15 a 19 anos, a média foi $1,57 \pm$ 0,4 , sendo o controle classificado como ruim.

$\mathrm{Na}$ tabela 2, observa-se que, independentemente do controle glicêmico, a maior parte dos diabéticos usava apenas edulcorante para adoçar os alimentos. Entretanto, a proporção de indivíduos com controle ruim foi significantemente maior entre aqueles que associavam sacarose e edulcorante e os que utilizavam apenas sacarose como agente adoçante $(68 \%$ e $100 \%$, respectivamente). Na avaliação do lanche escolar, observou-se que, entre os que levavam lanche de casa, houve predomínio de indivíduos com bom controle (53,3\%). Entre os entrevistados que consumiam a merenda oferecida na escola, compravam lanche ou não faziam o lanche, houve predomínio de indivíduos com controle ruim $(53,8 \%, 64,7 \%, 66,7 \%$, respectivamente). Dos lanches que os pacientes levavam para a escola, os mais citados foram: biscoitos sem recheio (64\%), frutas $(50 \%)$, suco artificial sem sacarose $(36,4 \%)$, pães $(18,2 \%)$, salgadinho tipo chips $^{\circledR}(15,2 \%)$, iogurte sem sacarose $(13,6 \%)$ e leite (11\%).

Tabela 2. Associação entre tipo de agente adoçante, lanche escolar e controle glicêmico dos participantes do estudo

\begin{tabular}{|c|c|c|c|c|}
\hline & \multicolumn{3}{|c|}{ Controle glicêmico } & \multirow{2}{*}{ p } \\
\hline & Bom & Regular & Ruim & \\
\hline $\begin{array}{l}\text { Uso do agente } \\
\text { adoçante }(n=139)\end{array}$ & & & & $0,004^{b}$ \\
\hline $\begin{array}{l}\text { Usa sacarose e } \\
\text { edulcorante }(n=25)\end{array}$ & $4(16,0 \%)$ & $4(16,0 \%)$ & $17(68,0 \%)$ & \\
\hline $\begin{array}{l}\text { Usa apenas edulcorante } \\
(n=109)\end{array}$ & $49(45,0 \%)$ & $21(19,3 \%)$ & $39(35,7 \%)$ & \\
\hline $\begin{array}{l}\text { Usa apenas sacarose } \\
(\mathrm{n}=3)\end{array}$ & $0,0 \%$ & $0,0 \%$ & $3(100,0 \%)$ & \\
\hline Outros $(n=2)$ & $0,0 \%$ & $0,0 \%$ & $2(100,0 \%)$ & \\
\hline $\begin{array}{l}\text { Lanche da escola } \\
(n=131)\end{array}$ & & & & $0,007^{\mathrm{a}}$ \\
\hline Merenda gratuita $(n=39)$ & $14(36 \%)$ & $4(10,2 \%)$ & $21(53,8 \%)$ & \\
\hline Lanche de casa $(n=60)$ & $32(53,3 \%)$ & $13(21,7 \%)$ & $15(25 \%)$ & \\
\hline Compra lanche $(n=17)$ & $1(5,9 \%)$ & $5(29,4 \%)$ & $11(64,7 \%)$ & \\
\hline Não faz lanche $(n=15)$ & $4(26,6 \%)$ & $1(6,7 \%)$ & $10(66,7 \%)$ & \\
\hline
\end{tabular}

Não foi observada associação estatisticamente significante entre regularidade de horários das refeições, número de refeições, hábito de "beliscar" e controle glicêmico dos participantes do estudo.

A média do IG e da CG da dieta da população estudada foi de $58,2 \pm 4,3$ e $138,6 \pm 37,5$, respectivamente. Os indivíduos com bom controle glicêmico ingeriam dieta com média do IG significantemente menor, 54,8 \pm 2,7 , do que aqueles que apresentaram controle glicêmico regular $60,1 \pm 3,8$ e ruim $60,3 \pm 4,1(\mathrm{p}=0,000)$. A CG da dieta também foi significantemente menor no grupo com bom controle glicêmico $(118,3 \pm 29)$ em relação aos grupos com controle regular e ruim, $142,5 \pm 27,3$ e $153,7 \pm 40,7$, respectivamente $(\mathrm{p}=0,000)$ (Tabela 3 ).

A dieta dos participantes do estudo continha, em média, $30,0 \pm 7,3 \mathrm{~g}$ de fibras. Não houve associação entre ingestão de fibras e controle glicêmico.

Foi observada associação significante entre ingestão de proteínas, gordura saturada e controle glicêmico, ou seja, os indivíduos com bom controle glicêmico apresentaram ingestão significantemente maior de proteínas e menor de gordura saturada em relação aos indivíduos com controle glicêmico ruim $(\mathrm{p}=0,016)$ (Tabela 3$)$.

Ao avaliar a contribuição dos diferentes grupos de alimentos para o controle glicêmico da população estudada, observou-se associação estatisticamente significante apenas em relação aos cereais, sendo sua ingestão menor no grupo de indivíduos com bom controle glicêmico em relação aos que tinham controle regular e ruim (Tabela 4).

Tabela 3. Média $\pm \mathrm{DP}$ do teor de macronutrientes, ácidos graxos, colesterol e fibras das dietas consumidas pelos participantes do estudo, distribuídos por grau de controle glicêmico

\begin{tabular}{lcccc}
\hline \multirow{2}{*}{ Nutrientes } & \multicolumn{3}{c}{ Controle glicêmico } & \\
\cline { 2 - 4 } & $\begin{array}{c}\text { Bom } \\
(\mathbf{n = 5 3 )}\end{array}$ & $\begin{array}{c}\text { Regular } \\
(\mathbf{n = 2 5 )}\end{array}$ & $\begin{array}{c}\text { Ruim } \\
(\mathbf{n = 6 1 )}\end{array}$ & $\mathbf{p}^{\mathbf{a}}$ \\
\hline \% Carboidrato & $53,5 \pm 4,7$ & $54,7 \pm 5,1$ & $55 \pm 5,1$ & 0,268 \\
\% Carboidrato + mono & $62,0 \pm 4,0$ & $63 \pm 4,2$ & $63,3 \pm 4,1$ & 0,281 \\
\% Proteína & $16,4 \pm 2,6^{\mathrm{b}}$ & $15,4 \pm 2,8$ & $15,0 \pm 2,6^{\mathrm{c}}$ & 0,016 \\
\% Gordura & $30,2 \pm 3,5$ & $29,7 \pm 3,7$ & $29,9 \pm 3,6$ & 0,818 \\
\% Saturada & $8,9 \pm 1,7^{\mathrm{b}}$ & $9,6 \pm 1,9$ & $10,1 \pm 2^{\mathrm{c}}$ & 0,031 \\
\% Monoinsaturada & $8,6 \pm 1,4$ & $8,3 \pm 1,4$ & $8,3 \pm 1,6$ & 0,609 \\
\% Poliinsaturada & $6,6 \pm 1,4$ & $7,1 \pm 1,2$ & $6,6 \pm 1,2$ & 0,210 \\
Colesterol (mg) & $221,4 \pm 70,0$ & $205,2 \pm 64$ & $223,6 \pm 94,3$ & 0,617 \\
Fibra (g/1.000 kcal) & $16,3 \pm 3,9$ & $15,94 \pm 1,77$ & $15,16 \pm 2,89$ & 0,136 \\
Índice glicêmico & $54,8 \pm 2,7^{d}$ & $60,1 \pm 3,8^{\mathrm{e}}$ & $60,3 \pm 4,1^{\mathrm{e}}$ & 0,000 \\
Carga glicêmica & $118,3 \pm 29,8^{\mathrm{d}}$ & $142,5 \pm 27,3^{\mathrm{e}}$ & $153,7 \pm 40,7^{\mathrm{e}}$ & 0,000 \\
\hline
\end{tabular}

a: teste ANOVA; b, c: diferem entre si; d, e: diferem entre si. DP: desvio-padrão. 
Tabela 4. Média $\pm D P$ da participação relativa dos grupos de alimentos no consumo alimentar total dos participantes do estudo, distribuídos pelo grau de controle glicêmico apresentado

\begin{tabular}{lcccc}
\hline & \multicolumn{3}{c}{$\begin{array}{c}\text { Controle } \\
\text { glicêmico }\end{array}$} & \multirow{2}{*}{$\mathbf{p}^{\mathbf{a}}$} \\
\cline { 2 - 4 } Grupos de alimentos & $\begin{array}{c}\text { Bom } \\
(\mathbf{n = 5 3 )}\end{array}$ & $\begin{array}{c}\text { Regular } \\
(\mathbf{n = 2 5 )}\end{array}$ & $\begin{array}{c}\text { Ruim } \\
(\mathbf{n = 6 1 )}\end{array}$ & \\
\hline Laticínios (\%) & $19,9 \pm 12,4$ & $21,3 \pm 16,3$ & $23,5 \pm 15,7$ & 0,048 \\
Leguminosas (\%) & $14,9 \pm 5,7$ & $13,0 \pm 4,2$ & $12,5 \pm 5,2$ & 0,949 \\
Carnes (\%) & $12,0 \pm 4,5$ & $11,0 \pm 3,4$ & $9,5 \pm 4,3$ & 0,954 \\
Hortaliças (\%) & $9,2 \pm 6,4$ & $7,9 \pm 4,1$ & $7,9 \pm 4,3$ & 0,342 \\
Frutas (\%) & $18,0 \pm 10,5$ & $15,8 \pm 10,9$ & $14,8 \pm 9,0$ & 0,677 \\
Cereais (\%) & $23,1 \pm 7,9^{b}$ & $27,8 \pm 7,0^{c}$ & $29,0 \pm 8,8^{c}$ & 0,000 \\
Gorduras/guloseimas (\%) & $2,8 \pm 2,6$ & $3,1 \pm 1,8$ & $3,0 \pm 2,4$ & 0,594 \\
\hline
\end{tabular}

a: teste ANOVA; b, c: diferem entre si. DP: desvio-padrão.

A renda e o grau de escolaridade dos pais não influenciaram o controle glicêmico da população estudada.

Não foi observada associação estatisticamente significante entre a dose de insulina e o controle glicêmico. No entanto, o controle glicêmico ruim foi observado em $87 \%$ dos indivíduos que não aplicavam a insulina conforme prescrição médica e em $34,5 \%$ dos que aplicavam corretamente $(\mathrm{p}=0,000)$. A não aplicação da dose de insulina prescrita não foi afetada pela renda ou pelo grau de escolaridade dos pais. A monitorização da glicemia também influenciou o controle glicêmico desse grupo. Dos indivíduos que não monitorizavam a glicemia $(\mathrm{n}=29), 62,1 \%$ apresentavam controle glicêmico ruim. Entre os que monitorizavam a glicemia 1 a 2 vezes/dia $(\mathrm{n}=48)$ ou 3 a 4 vezes $/$ dia $(\mathrm{n}=56)$, a proporção de indivíduos com controle glicêmico ruim foi significantemente menor $(50 \%$ e $26,8 \%$, respectivamente; $\mathrm{p}=0,022)$. Houve predomínio dos indivíduos com idade entre 11 e 19 anos entre os que não aplicavam insulina conforme a prescrição e do grupo de 15 a 19 anos entre os que não monitorizavam a glicemia $(\mathrm{p}<0,05)$.

\section{DISCUSSÃO}

A importância da adoção de hábitos alimentares saudáveis por indivíduos diabéticos tem sido apontada como medida relevante para o controle da doença e a prevenção das complicações agudas e crônicas (5). No entanto, verifica-se, ainda, escassez de estudos em que se avaliou o impacto dos hábitos alimentares no controle metabólico de crianças e adolescentes com DMl. Em estudo recente, observou-se que o hábito de tomar café da manhã e jantar regularmente associou-se ao controle glicêmico adequado nesses indivíduos (19). Em outro estudo, foi verificado que a realização de um maior número de refeições por dia favorece o controle glicêmico de adolescentes diabéticos, refletindo uma sincronia adequada entre insulina e dieta $(20)$.

Nesta casuística, o hábito de levar lanche de casa para a escola favoreceu o controle glicêmico da população estudada. Entre os alimentos mais citados como opções de lanche escolar levado de casa, constatou-se que houve predomínio dos alimentos sugeridos habitualmente para refeições intermediárias e, que se não consumidos em excesso, podem evitar o desequilíbrio glicêmico. Em contrapartida, a merenda gratuita e o lanche comprado na escola afetaram negativamente o controle, sugerindo inadequação na qualidade do lanche oferecido nas escolas públicas, que geralmente é composto por preparações ricas em calorias e, muitas vezes, em carboidratos e lípides (21). Considerando que a alimentação ideal da criança diabética corresponde à alimentação saudável de qualquer criança, seria desejável a reestruturação dos cardápios da merenda escolar e o controle da qualidade dos lanches vendidos nas cantinas. O consumo diário de refeições apresentando composição nutricional inadequada pode afetar o controle metabólico dos diabéticos, com as consequências já conhecidas.

O consumo habitual de sacarose afetou negativamente o controle glicêmico dos participantes do estudo. As novas diretrizes nutricionais para o $\mathrm{DM}$ não fazem restrição quanto ao tipo de carboidrato consumido. Segundo recomendações da ADA (5), a sacarose pode ser incluída na dieta do diabético dentro do contexto de uma alimentação saudável, podendo ser consumida em substituição a outros tipos de carboidratos. Neste estudo, a sacarose ingerida pelo grupo não foi quantificada. Uma possibilidade, no entanto, seria que a associação observada fosse devida ao consumo excessivo desse alimento. Assim, a ingestão de sacarose e de alimentos açucarados deve ser monitorada, pois, além de ser fonte concentrada de carboidratos, não fornece nenhum outro nutriente essencial ao organismo, podendo comprometer o controle glicêmico e, ainda, contribuir para o ganho excessivo de peso.

O impacto do carboidrato ingerido no controle glicêmico dos pacientes pôde ser visualizado a partir da estimativa do IG e da CG. O consumo de dieta com IG/CG mais baixos esteve relacionado ao melhor controle glicêmico da população estudada. Os resultados 
sugerem que tanto a qualidade (IG) quanto a quantidade $(C G)$ de carboidrato ingerido afetaram a resposta glicêmica. O mecanismo pelo qual o consumo de dietas de baixo IG e CG pode interferir no controle glicêmico não está totalmente elucidado. Apesar disso, a influência positiva do IG no controle glicêmico de indivíduos com DM foi confirmada em vários estudos (22-25). Tem sido sugerido que o efeito benéfico de uma dieta de baixo IG esteja relacionado à redução do pico pósprandial da glicose e, consequentemente, da demanda de insulina (26). No presente estudo, entre os grupos com controle glicêmico regular e ruim não houve diferença estatisticamente significante entre as médias do IG e da CG da dieta ingerida, sugerindo que outros fatores contribuíram para o grau de controle observado. Deve ser considerado que a relação entre consumo de dieta com IG/CG mais baixos e melhor controle glicêmico da população estudada ocorreu na ausência de ajuste da dose de insulina pré-prandial ao total de carboidratos consumidos nas refeições, que é uma das estratégias nutricionais que pode contribuir para melhorar o controle glicêmico. O menor consumo de alimentos do grupo dos cereais influenciou positivamente o controle glicêmico da população estudada. Considerando que neste grupo encontravam-se as principais fontes de carboidratos da dieta e a maioria $(76,5 \%)$ dos alimentos com alto IG incluídos no QQFA, presumese que seu menor consumo pode ter contribuído para reduzir o IG e a CG da dieta dos indivíduos com bom controle glicêmico.

Foi detectada associação direta entre ingestão de gordura saturada e controle glicêmico ruim. Resultado semelhante foi observado em estudo envolvendo 48 meninas diabéticas tipo 1 , com idade entre 10 e 19 anos (27). Em outros estudos, essa associação não foi observada $(28,29)$. Entretanto, nestes dois últimos estudos, a ingestão de gordura saturada superou o limite máximo da recomendação adotada para o grupo como um todo, dificultando a observação dessa associação. Muitos aspectos da relação entre ingestão de lípides e controle glicêmico ainda necessitam ser esclarecidos. Em indivíduos saudáveis, estudos demonstraram associação entre ingestão de dieta rica em gordura saturada e redução da sensibilidade à ação da insulina $(30,31)$. Os mecanismos pelos quais a natureza da gordura pode interferir na sensibilidade à insulina não estão totalmente entendidos. Alguns autores acreditam que a alta ingestão de gordura saturada provoque uma modificação do perfil lipídico da membrana das células, tornando a membrana fosfolipídica mais saturada e, consequentemente, mais resistente à ação da insulina (32).

Embora o percentual de proteínas ingeridas estivesse dentro do recomendado em todos os grupos, indivíduos com bom controle glicêmico apresentaram maior ingestão desse nutriente em relação aos de controle ruim. Em indivíduos saudáveis, o consumo de dietas com alto teor de proteínas pode atenuar a resposta glicêmica por aumentar a secreção de insulina (33). No entanto, em indivíduos com DMl essa associação ainda não está bem estabelecida, pois há comprometimento da secreção da insulina. Assim, mais estudos são necessários para explicar tal associação.

Observou-se baixa prevalência de excesso de peso neste grupo, diferentemente do que vem sendo relatado em vários outros estudos envolvendo crianças e adolescentes com DMl $(19,34,35)$.

Porém, todos os indivíduos com sobrepeso apresentavam controle glicêmico ruim, o que poderia estar também relacionado à resistência insulínica e à redução da efetividade da insulina administrada, comumente observada em indivíduos com excesso de peso (1).

A dificuldade em aderir a rotina de aplicação de insulina e monitorização da glicemia afetou negativamente o controle glicêmico da população estudada. As mudanças na rotina diária do paciente e de sua família vão além dos aspectos físicos, abrangendo também aspectos psicossociais. $\mathrm{O}$ tratamento pode gerar frustrações pela redução da autonomia pessoal, o que pode ser um problema principalmente na adolescência, fase caracterizada pela dificuldade em aceitar regras e orientações terapêuticas.

Como conclusão, verificou-se que o consumo frequente de sacarose e merenda gratuita afetou negativamente o controle glicêmico de crianças e adolescentes diabéticos. Já o consumo de dietas com maior teor de proteína e menor teor de gordura saturada e com IG/CG mais baixos contribuiu para o melhor controle dos indivíduos estudados.

Esses dados apontam para a necessidade de intensificar o aconselhamento nutricional dos diabéticos nessa faixa etária, orientando para o consumo de dieta nutricionalmente mais adequada com redução do IG/ CG. Adicionalmente, torna-se necessário um trabalho conjunto com os responsáveis pelo planejamento e pela elaboração dos cardápios da merenda gratuita oferecida nas escolas públicas, para sua revisão, visto que o consumo diário de refeições de composição nutricional inadequada pode comprometer a saúde das crianças e, especialmente, o controle metabólico dos diabéticos.

Declaração: os autores declaram não haver conflitos de interesse científico neste estudo. 


\section{REFERÊNCIAS}

1. Barker JM, Goehrig SH, Barriga K, Hoffman M, Slover R, Eisenbarth GS, et al. Clinical characteristics of children diagnosed with type 1 diabetes through intensive screening and follow-up. Diabetes Care. 2004;27(6):1399-404.

2. Ministério da Saúde. Organização Pan-Americana da Saúde. Avaliação do Plano de Reorganização da Atenção à Hipertensão Arterial e ao Diabetes Mellitus no Brasil, Brasília: MS; 2004.

3. The Diabetes Control and Complications Trial Research Group. The effect of intensive treatment of diabetes on development and progression of long-term complications in insulin-dependent diabetes mellitus. N Engl J Med. 1993;329:977-86.

4. The Diabetes Control and Complications Trial/Epidemiology of Diabetes Interventions and Complications (DCCT/EDIC) Study Research Group. Intensive diabetes treatment and cardiovascular disease in patients with type 1 diabetes. $\mathrm{N}$ Engl $\mathrm{J}$ Med. 2005;353:2643-53.

5. American Diabetes Association. Nutrition recommendations and interventions for diabetes. A position statement of the American Diabetes Association. Diabetes Care. 2008;31 Suppl 1:S61-74.

6. Jellife DB. Evaluacion del estado de nutrición de la comunidad. Ginebra: Organizacion Mundial de la Salud; 1968.

7. Bray GA, Grey DS. Obesity. Part I - Pathogenesis. West J Med. 1988;149(4):429-41.

8. Himes $\mathrm{J} \mathrm{H}$. Challenges of accurately measuring and using BMI and other indicators of obesity in children. Pediatrics. 2009;124 Suppl:S3-S22.

9. Monteiro JBR, Esteves E. Diet Pro, versão 4.0: Sistema de suporte à avaliação nutricional e prescrição de dietas. A Agromídia Software; 2001. [CD-ROM]

10. Chase HP, Jackson SL, Hoops RS, Cockerham PG, O' Brien D. Glucose control and the renal and retinal complications of insulindependent diabetes. JAMA. 1989;261(8):1155-60.

11. Ribeiro $A B$, Cardoso $M A$. Construção de um questionário de freqüência alimentar como subsídio para programas de prevenção de doenças crônicas não transmissíveis. Rev Nutr. 2002;15(2):239-45.

12. Monteiro JP, Pfrimer K, Tremeschin MH, Molina MC, Chiarello P. Consumo alimentar. Visualizando porções. Rio de Janeiro: Guanabara Koogan; 2007.

13. Tabela brasileira de composição de alimentos/NEPA-Unicamp, versão II. Campinas: NEPA-Unicamp; 2006.

14. Philippi ST.Tabela de composição de alimentos: suporte para decisão nutricional. 2.ed. São Paulo: Roca; 2005.

15. Pacheco M. Tabela de equivalentes, medidas caseiras e composição química dos alimentos. Rio de Janeiro: Livraria e Editora Rubio; 2006.

16. Philippi ST, Latterza AR, Cruz ATR, Ribeiro LC. Pirâmide alimentar adaptada: guia para escolha dos alimentos. Rev Nut. 1999;12(1):65-80.

17. Wolever TMS, Jenkins DJA. The use of glycemic index in predicting the blood response to mixed meals. Am J Clin Nutr. 1986;43(1):167-72.

18. Foster-Powell $\mathrm{K}$, Holt SH, Brand-Miller JC. International table of glycemic index and glycemic load values. Am J Clin Nutr. 2002;76(1):5-56.
19. Orbey NC, Margeirsdottir HD, Brunborg C, Andersen LF, DahlJorgensen $\mathrm{K}$. The influence of dietary intake and meal pattern on blood glucose control in children and adolescents using intensive insulin treatment. Diabetologia. 2007;50(10):2044-51.

20. Virtanen SM. Metabolic control and diet in finnish diabetic adolescents. Acta Paediatr. 1992;81:239-43.

21. Seyffarth AS. Abordagem nutricional em diabetes mellitus. Brasília: Ministério da Saúde; 2000. p. 155.

22. Buyken AE, Toeller M, Heitkamp G, Karamanos B, Rottiers R, Muggeo $M$, et al. Glycemic index in the diet of European outpatients with type 1 diabetes: relations to glycated hemoglobin and serum lipids. Am J Clin Nutr. 2001;73:574-81.

23. Opperman AM, Venter CS, Oosthuizen W, Thompson RL, Vorster $\mathrm{HH}$. Meta-analysis of the health effects of using the glycemic index in meal-planning. Br J Nutr. 2004;92:367-81.

24. Gilbertson HR, Brand-Miller JC, Thorburn AW, Evans S, Chondros P. The effect of flexible low glycemic index dietary advice versus measured carbohydrate exchange diets on glycemic control in children with type 1 diabetes. Diabetes Care. 2001;24(7):1137-43.

25. Burani J, Longo PJ. Low-glycemic index carbohydrates: an effective behavioral change for glycemic control and weight management in patients with type 1 and 2 diabetes. Diabetes Educ. 2006;32(1):78-88.

26. Jenkins DJ, Franceschi S, Hamidi M, Marchie A, Jenkins AL, Axelsen M. Glycemic index: overview of implications in health and disease. Am J Clin Nutr. 2002;76(suppl):S266-S73.

27. Pietiläinen $K H$, Virtanen $S M$, Rissanen $A$, Rita $H$, Mäenpää J. Diet, obesity and metabolic control in girls with insulin dependent diabetes mellitus. Arch Dis Child. 1995;73:398-402.

28. Forsander G, Malmodin B, Eklund C, Persson B. Relationship between dietary intake in children with diabetes mellitus type 1 their management at diagnosis, social factors, anthropometry and glycaemic control. Scand J Nutr. 2003;47(2):75-84.

29. Wiltshire EJ, Hirte C, Couper JJ. Dietary fats do not contribute to hyperlipidemia in children and adolescents with type 1 diabetes. Diabetes Care. 2003;26(5):1356-61.

30. Pérez-Jiménez F, López-Miranda J, Pinillos MD, Gómez P, Paz-Rojas $\mathrm{P}$, Montilla $\mathrm{C}$, et al. A Mediterranian and a high-carbohydrate diet improve glucose metabolism in healthy young persons. Diabetologia. 2001;44:2038-43.

31. Vessby B, Uusitupa M, Hermansen K, Riccardi G, Rivellese AA, Tapsell LC, et al. Substituing dietary saturated for monounsatured fat impairs insulin sensitivity in healthy men and women: the KANWU Study. Diabetologia. 2001;44:312-19.

32. McAuley K, Mann J. Nutritional determinants of insulin resistance. J Lipid Res. 2006;47:1668-76.

33. Moghaddam E, Vogt JA, WoleverTMS. The effects of fat and protein on glycemic responses in nondiabetic humans vary with waist circumference, fasting plasma insulin, and dietary fiber intake. J Nutr. 2006;136:2506-11.

34. Moraes CM, Portella RB, Pinheiro VS, Oliveira MMS, Fuks AG, Cunha $E F$, et al. Prevalência de sobrepeso e obesidade em pacientes com diabetes mellitus tipo 1. Arq Bras Endocrinol Metabol. 2003;47:677-83.

35. Arcanjo CL, Piccirillo LJ, Machado IV, Andrade Jr CRM, Clemente EL, Gomes MB. Avaliação de dislipidemia e de índices antropométricos em pacientes com diabetes mellitus tipo 1. Arq Bras Endocrinol Metabol. 2005;49:951-8. 